\title{
Combustion of a neutron star into a strange quark star: The neutrino signal
}

\author{
Giuseppe Pagliara $^{(a)}$, Matthias Herzog ${ }^{(b)}$ and Friedrich K. Röpke ${ }^{(\mathrm{c})}$ \\ ${ }^{(a)}$ Dipartimento di Fisica e Scienze della Terra dell'Università di Ferrara \\ and INFN Sezione di Ferrara, Via Saragat 1, I-44100 Ferrara, Italy \\ (b) Max-Planck-Institut für Astrophysik, Karl-Schwarzschild-Strasse 1, D-85741 Garching, Germany and \\ ${ }^{(c)}$ Institut für Theoretische Physik und Astrophysik, Universität Würzburg, \\ Emil Fischer-Strasse 31, D-97074 Würzburg, Germany
}

\begin{abstract}
There are strong indications that the process of conversion of a neutron star into a strange quark star proceeds as a strong deflagration implying that in a few milliseconds almost the whole star is converted. Starting from the three-dimensional hydrodynamic simulations of the combustion process which provide the temperature profiles inside the newly born strange star, we calculate for the first time the neutrino signal that is to be expected if such a conversion process takes place. The neutrino emission is characterized by a luminosity and a duration that is typical for the signal expected from protoneutron stars and represents therefore a powerful source of neutrinos which could be possibly directly detected in case of events occurring close to our Galaxy. We discuss moreover possible connections between the birth of strange stars and explosive phenomena such as supernovae and gamma-ray-bursts.
\end{abstract}

PACS numbers: 21.65.Qr,26.60.Dd

\section{INTRODUCTION}

Under the hypothesis that the true ground state of nuclear matter is cold strange quark matter [1-5] it is likely that compact stars exist that are composed almost entirely of quark matter, the so-called strange stars. In turn, the birth of these stellar objects can be thought of as a decay of a metastable neutron star into a more bound configuration with a consequent huge release of energy, of the order of $10^{53} \mathrm{erg}$. If released in a short time period, the fascinating possibility exists that this energy source is associated with the most extreme explosions of the Universe, i.e. supernovae ( $\mathrm{SNe}$ ) and gammaray-bursts (GRBs). The strange quark matter hypothesis could possibly be proven also by detecting the gravitational wave signal associated with the merger process of two strange stars as pointed out in recent simulations [6, 7].

There are several issues related to the conversion process that have been investigated in the past. A first problem concerns the initial seed of strange quark matter which then triggers the conversion: Several studies have addressed the thermal and quantum nucleation of the first drop of quark matter, and different possible scenarios have been proposed [8 16]. A second and more complicated question concerns the time needed for the conversion of the whole star. In the first study addressing this issue [17], a laminar conversion front was assumed with a resulting time needed for the conversion of up to 100s. Such a slow process would provide a very faint neutrino signal and thus not be relevant from the phenomenological point of view. On the other hand, it was pointed out and estimated that hydrodynamical instabilities can substantially increase the velocity of the conversion front [18, 19] leading to a regime, in most of the cases, of strong deflagration [20, 21]. In Ref. 21], in particular, many different equations of state (includ- ing hyperons, color superconductivity and mixed phases) have been tested, also considering the effects of keeping the flavor composition of matter fixed during the combustion, and no case has been found in which the conversion is so rapid to turn itself into a detonation process. Also, the temperature inside the newly born star has been estimated with resulting central temperatures of the order of tens of $\mathrm{MeV}$. Finally, a numerical study on this problem has recently provided more quantitative results on the conversion process 22]: Three-dimensional hydrodynamic simulations have been conducted under the hypothesis that the conversion of a hadronic neutron star into a strange quark star is a combustion process. The combustion turned out to be turbulent due to the growth of buoyancy instabilities. Turbulence enhances the burning velocity considerably, leading to short conversion time scales of a few milliseconds. Moreover, it has been found that, even if strange quark matter is absolutely stable, the hydrodynamic combustion process is not able to burn the whole neutron star; a sizable layer survives which then probably converts on a longer time scale.

Finally, a third problem that has never been addressed in detail concerns how the huge energy of the conversion process is emitted by the star. Clearly, the heat released by the formation of strange quark matter is dissipated via a strong neutrino emission, very similar to the emission of a protoneutron star. In this paper, starting from the hydrodynamic simulations of the conversion process, we compute for the first time the neutrino signal that is to be expected if a neutron star decays into a strange quark star (some simple estimates have been presented in [23]). In particula,r we present results for the diffusion of the neutrinos inside the newly born quark star and the consequent cooling of the star. As expected, both the luminosity and the duration of the neutrino signal are very similar to the ones of protoneutron stars. The birth 
of a strange star is thus phenomenologically very relevant, because the corresponding neutrino signal could be detected by the presently available neutrino telescopes if it occurs close enough to our Galaxy. Moreover, the formation of strange quark matter in a magnetar could represent a possible extension of the so-called protomagnetar model of GRBs 24] which, as we will discuss, might be able to explain some recent puzzling observations of GRBs.

The paper is organized as follows: In Sec. II we discuss the equations of state (EOSs) adopted for nucleonic matter and for strange quark matter. In particular, we will consider EOSs for strange quark matter that allow us to obtain a maximum mass for cold and catalyzed stellar configurations compatible with the $2 M_{\odot}$ limit [25]. In Sec. III, we review the procedure adopted to simulate the conversion process as done in Ref. 22], and we show the initial conditions for the neutrino diffusion calculations. In Sec. IV, we present and discuss the calculation of the process of diffusion of neutrinos within the newly born strange quark star. In Sec. V, after a discussion on the possible phenomenological implications of the process under study, we draw our conclusions.

\section{EQUATIONS OF STATE}

For the nucleonic matter EOS we use the table computed by Lattimer and Swesty (LS EOS) [26], which is based on a liquid drop model. To be consistent with the observation of a two solar mass (see below) neutron star, we apply an incompressibility modulus of $K=220 \mathrm{MeV}$. For reasons discussed in detail in [22], nucleonic matter EOSs of much higher stiffness, e.g. the relativistic mean field EOS by [27], could not be employed, because they do not allow for exothermic combustion. For the strange quark matter EOS we use, as customary, the MIT bag model with the inclusion of the perturbative QCD corrections [28, 29]. For this work, we neglect the effects related to the appearance of diquark condensates, as in the color-flavor-locking phase, for instance [30]. A detailed study on this possibility will be important: A second order phase transition from unpaired quark matter to gapped matter could indeed take place in the astrophysical object that we are considering, possibly providing interesting signatures on the neutrino signal [31]. Also, the change of the chemical composition of the star during deleptonization could lead to first-order phase transition between different quark phases [32]. Concerning the free parameters of the quark matter model, the discovery of a $2 M_{\odot}$ compact star 25] has significantly reduced its parameter space as shown in 33. We consider two sets of parameters, taken from 33], both allowing us to reach a maximum mass for strange quark stars of $2 M_{\odot}$. We fix the current mass of the strange quark to $m_{s}=100$ $\mathrm{MeV}$, and we then consider $B_{\text {eff }}^{1 / 4}=142 \mathrm{MeV}-a_{4}=0.9$ (set1) and $B_{\text {eff }}^{1 / 4}=141 \mathrm{MeV}-a_{4}=0.65$ (set2) where $B_{\text {eff }}$ and $a_{4}$ represent the effective bag constant and the coef-

\begin{tabular}{|l|l|l|l|l|l|}
\hline$M_{\odot}($ set 1$)$ & $\mathrm{a}$ & $\mathrm{b}$ & $\mathrm{c}$ & $\mathrm{d}$ & $\mathrm{e}$ \\
\hline 1.4 & 10.7 & 0.048 & 0.0025 & 39.1 & 18.4 \\
\hline 1.8 & 9.5 & 0.054 & 0.0023 & 43.8 & 18.9 \\
\hline
\end{tabular}

TABLE I: Parameters of the fit to the numerical results of temperature profiles.

ficient of the $\mu^{4}$ term in the pressure of the quark phase, respectively ( $\mu$ is the quark chemical potential), as in Ref. [34]. While the two sets provide the same maximum mass, they correspond to different values of the energy per baryon of the ground state of strange quark matter: for set1 $E / A=860 \mathrm{MeV}$ and for set2 $E / A=930$ $\mathrm{MeV}$; in turn, this implies that for set1 the EOS is soft and for set 2 stiff. Indeed, these two sets of parameters lie on the two-flavor and three-flavor lines, respectively, in Fig. 1 of 33 . The EOSs are expressed as tables with the baryon density and the temperature as independent variables. Finally, the chemical potentials of quarks and electrons are fixed by the beta equilibrium conditions. Since our starting configuration is a cold and catalyzed neutron star, we fix to zero the chemical potential of neutrinos (see discussion in the following).

\section{COMBUSTION SIMULATIONS AND INITIAL CONDITIONS FOR THE COOLING}

To simulate the combustion process we adopt the scheme already used in 22]. We solve the Euler equations by using a well-tested grid code that employs a finite volume discretization, the so-called piecewise parabolic method 35]. In this code, the conversion is described in a discontinuity approximation meaning that the hadronic and newly converted strange quark matter is separated by a conversion front. This conversion front is modeled by using a level-set method 36 38. Further references are given in [22]. In our simulations, general relativity effects are included only by using an effective relativistic gravitational potential based on the TolmanOppenheimer-Volkov equations. It has been estimated in Ref. [39] that general relativity effects and the rotation of the star could lead to qualitative differences in the combustion. Presently, a 3D hydrodynamic simulation including also the before-mentioned effects is computationally quite challenging, but it is of course an important outlook for our study.

The conversion process is triggered by a strange quark matter seed in the center of a hadronic neutron star. Numerically, at the start of the simulation a volume in the center is converted instantly; the conversion front subsequently propagates outwards as a deflagration wave. The initial, laminar conversion velocity is based on detailed flame calculations of [40]. Turbulent conversion velocities are calculated by means of a sophisticated subgrid scale turbulence model [41, 42].

Except for the EOS for strange quark matter, we use 
exactly the same initial setup as in [22]. We simulate one octant of a neutron star in three dimensions and apply a resolution of 128 grid cells per dimension on a moving hybrid grid which provides optimal resolution in the regions of interest. This resolution is sufficient for our purpose, as was discussed in [22], where also further details of our initial setup can be found.

We have performed the simulations of the combustion by using the previously described strange quark matter and nucleonic matter EOSs and for two values of the mass of the initial neutron star configuration: 1.4 and $1.8 M_{\odot}$.

In Fig. 1, we illustrate the conversion process with snapshots taken at four different instants in time for a $1.4 M_{\odot}$ neutron star (set1). While the soft EOS obtained with set1 allows for a quite successful conversion of the star, for the stiff EOS obtained with set2, the requirement of having an exothermic process does not allow the combustion. The neutron star cannot be converted - at least within our framework.

In Fig. 2, we show the results for the temperature profiles as a function of pressure obtained from the combustion simulations. At the center of the star the temperature reaches quite high values, $40-50 \mathrm{MeV}$, and it drops steeply at the interface between the burned material and the unburned one. Together with the numerical results, indicated by dots and boxes, in Fig. 2 we show parametrizations of the temperature profiles that we will use as initial conditions for the neutrino diffusion calculation. The parametrization reads $T=a \operatorname{Arctan}((P-b) / c)+d P+e$, where $T$ and $P$ are temperature and pressure, respectively, and the parameters $a, b, c, d, e$ are obtained by fitting the numerical results (see Table 1 for their numerical values).

\section{COOLING OF THE STRANGE QUARK STAR}

The combustion process lasts typically a few milliseconds. On the other hand, the diffusion of neutrinos occurs on typical time scales of the order of tens of seconds. It is therefore a reasonable approximation to consider the two processes separately: One can use the output of the combustion simulation as an initial condition for the diffusion simulation. The diffusion approximation, usually adopted for the study of the evolution of protoneutron stars, is based on the assumption that neutrinos are locally in chemical and thermal equilibrium with baryonic matter and that their motion is driven by the gradients of chemical composition and temperature within the star. This approximation is valid if the neutrino's mean free path is much smaller than the size of the star, which turns out to be the case in protoneutron star matter and also in the system we are studying here. Of course, a better but much more complex approach would be to use a Boltzmann transport code [43, 44], which must be necessarily used in supernova simulations in which neutrinos propagate through very low density material in the outer layers of the progenitor star.

There is an important difference between protoneutron star matter and the matter after the combustion of a neutron star into a quark star. In the first case, apart from the high temperature, matter is also lepton rich $\left(Y_{L} \sim 0.4\right)$, and one can define a flux of net lepton number which diffuses inside the star and drives the deleptonization. After this process the stellar matter will be in beta equilibrium. The energy flux driven by neutrinos determines instead the cooling of the protoneutron star (see the simulations of protoneutron star evolution within the diffusion approximation in Refs. 32, 45 50]).

In our case, before the combustion, the matter of the neutron star was already in beta equilibrium, and the formation of the quark phase leads mainly to a strong reheating of matter. We can thus assume that the most important process for the evolution of the newly born quark star is the diffusion of the neutrino energy and we will use only the diffusion equation associated with the transport of energy [69].

An important point must be emphasized here: Within the hydrodynamical description of the combustion process, the conservation of the total lepton number is not imposed. The conversion indeed involves the beta stable nucleonic phase and the beta stable quark phase but the electron fraction in the nucleonic phase although small, $Y_{e} \sim 0.1$ at the center of the star, is much larger than the electron fraction within the quark phase (typically of the order of $10^{-5}$ by using an estimate for the electron chemical potential to be $\left.\mu_{e}=m_{s}^{2} / 4 \mu[30]\right)$. In principle, to impose lepton number conservation, one would need to add another conservation equation to the hydrocode and use a table for strange quark matter with another independent variable, i.e. the electron fraction. Clearly, such a procedure would lead to a different initial condition for the diffusion calculation.

Let us discuss now the equations that we solve to simulate the diffusion process. Apart from the cooling of the star caused by neutrino diffusion, one has also to consider the readjustment of the structure of the star due to the decreasing of the temperature. In the standard procedure, the diffusion equation is coupled with the structure equations which provide, at each time step, the hydrostatic configuration of the star. The energy diffusion equation and the structure equations read [46]

$$
\begin{aligned}
\frac{\mathrm{d}}{\mathrm{dt}} \frac{\epsilon_{t o t}}{n_{b}}+P \frac{\mathrm{d}}{\mathrm{dt}} \frac{1}{n_{b}} & =-\frac{\Gamma}{n_{b} r^{2} \mathrm{e}^{\Phi}} \frac{\partial}{\partial r}\left(\mathrm { e } ^ { 2 \Phi } r ^ { 2 } \left(F_{\epsilon, \nu_{e}}\right.\right. \\
& \left.\left.+F_{\epsilon, \nu_{\mu}}\right)\right) \\
\frac{d P}{d r} & =-\left(P+\epsilon_{t o t}\right) \frac{m+4 \pi r^{3} P}{r^{2}-2 m r} \\
\frac{d m}{d r} & =4 \pi r^{2} \epsilon_{t o t} \\
\frac{d a}{d r} & =\frac{4 \pi r^{2} n_{b}}{\sqrt{1-2 m / r}} \\
\frac{d \Phi}{d r} & =\frac{m+4 \pi r^{3} P}{r^{2}-2 m r}
\end{aligned}
$$




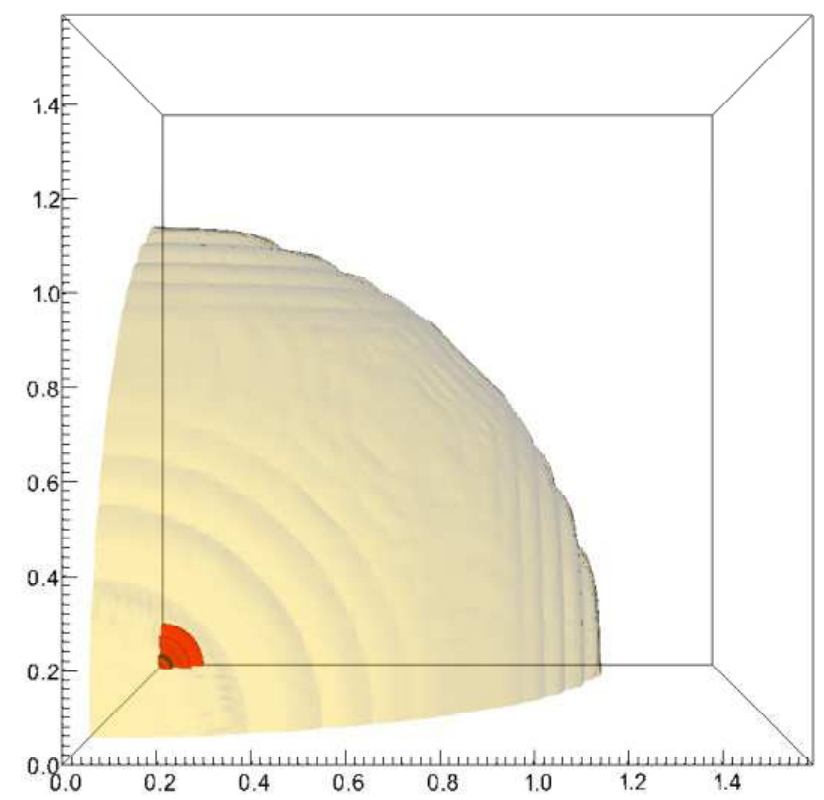

(a) $t=0$

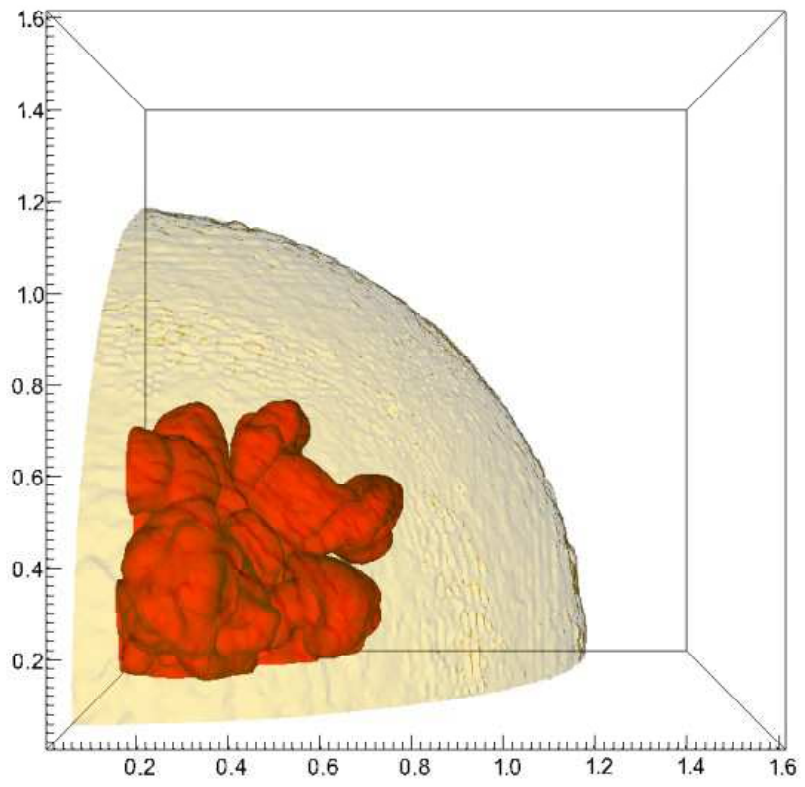

(c) $t=1.2 \mathrm{~ms}$

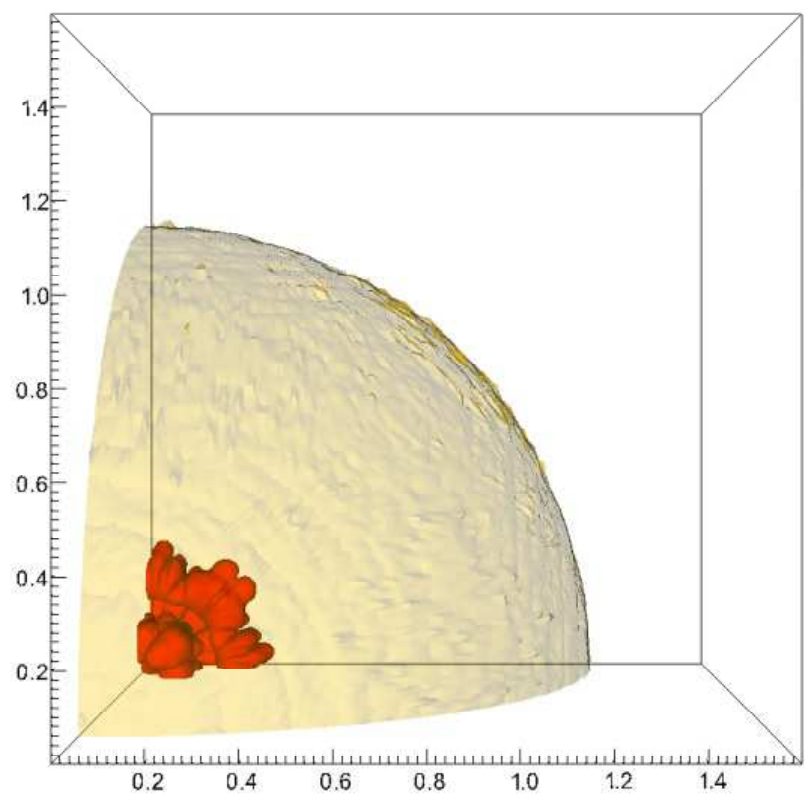

(b) $t=0.7 \mathrm{~ms}$

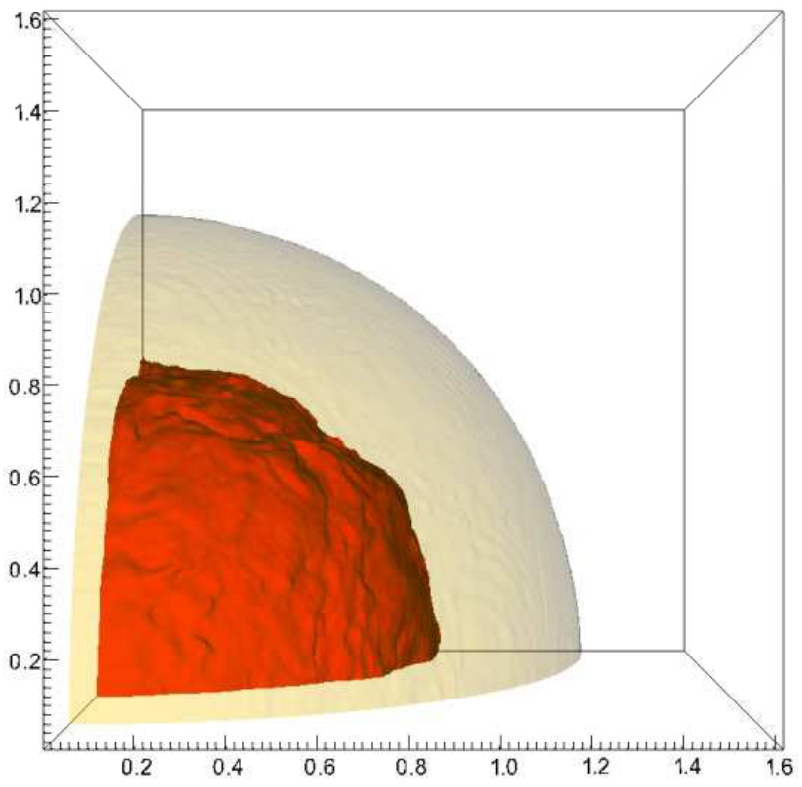

(d) $t=4.0 \mathrm{~ms}$

FIG. 1: (color online) Model: Set $1, M=1.4 M_{\odot}$. Conversion front (red) and surface of the neutron star (yellow) at different times $t$. Spatial units $10^{6} \mathrm{~cm}$.

where $\epsilon_{t o t}, n_{b}$, and $P$ are the total energy density, baryon density, and pressure of matter, respectively, $\Gamma=\sqrt{1-2 m(r) / r}$ where $m(r)$, is the gravitational mass enclosed within a radius $r, a$ is the enclosed baryonic mass and $\mathrm{e}^{\Phi}=\sqrt{g_{00}}$. The fluxes associated with the transport of energy by electron neutrinos and antineutri- nos and muon and tau neutrinos and antineutrinos are:

$$
\begin{aligned}
& F_{\epsilon, \nu_{e}}=-\frac{\lambda_{\epsilon, \nu_{e}}}{3} \frac{\partial \epsilon_{\nu_{e}}}{\partial r} \\
& F_{\epsilon, \nu_{\mu}}=-\frac{\lambda_{\epsilon, \nu_{\mu}}}{3} \frac{\partial \epsilon_{\nu_{\mu}}}{\partial r}
\end{aligned}
$$

where $\epsilon_{\nu_{e}}$ and $\epsilon_{\nu_{\mu}}$ are the energy densities of electron and muon neutrinos, respectively, while $\lambda_{\epsilon, \nu_{e}}$ and $\lambda_{\epsilon, \nu_{\mu}}$ 


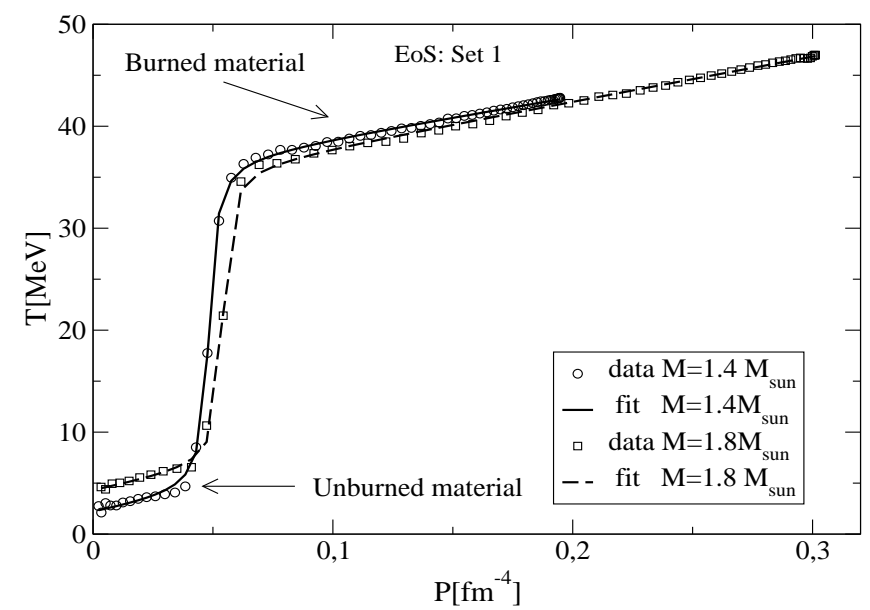

FIG. 2: Temperature as a function of the pressure after combustion. The two lines correspond to the numerical output of the simulations and the parametrization which are used for the diffusion calculations.

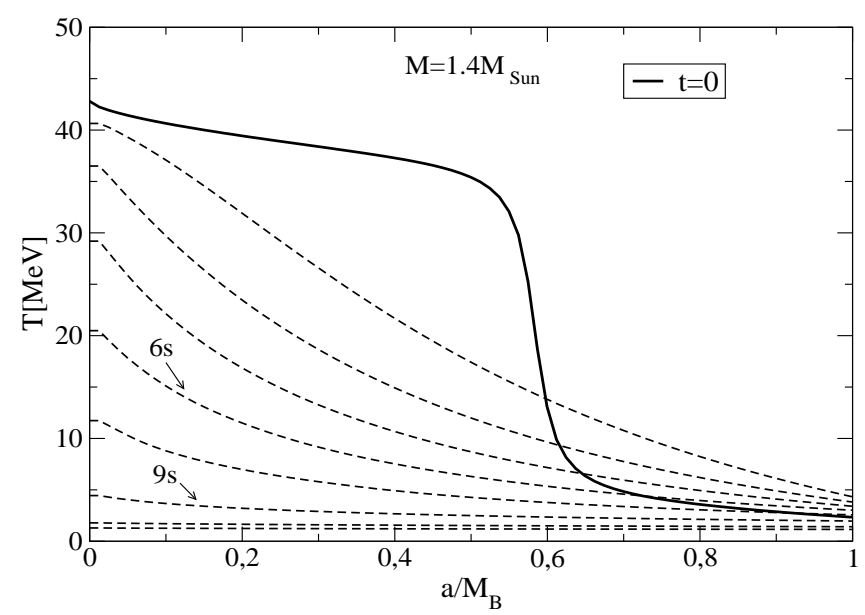

FIG. 3: Temperature evolution as a function of enclosed baryonic mass for a $1.4 M_{\odot}$ star.

represent spectral averages of the mean free paths of $\nu_{e}$ and $\nu_{\mu}$, respectively (the transport of $\nu_{\mu}$ and $\nu_{\tau}$ is treated as usual on the same footing).

To solve the equations previously introduced one needs, besides the initial condition on the temperature taken from the hydrodynamical simulations, the boundary conditions for the temperature at the center of the star and at the star's radius $R$. At $r=0$, one imposes that the energy fluxes $F_{\epsilon, i}$ vanish due to symmetry reasons. At the surface we assume, as in [46], the neutrino radiation to stream off into vacuum and impose the following conditions on the values of the fluxes: $F_{\epsilon, i}=\beta \epsilon_{i}$ where $\beta$ is a geometric factor which measures the degree of anisotropy of the radiation field at the surface. Values $\sim 0.5$ are usually considered for this parameter [46, 48].

Another important ingredient is of course the EOS.
Here a second main assumption for this calculation has to be done: The simulations of [22] have shown that, although in principle the whole star should convert into a strange quark star because of the hypothesis of absolutely stable quark matter, the combustion actually stops before the full conversion is reached. Most probably the conversion could go on via diffusion of strange quarks into the hadronic phase, as computed in [17], and of neutrons into the quark phase, a process that probably is exothermic as pointed out in [51]. We here assume that the conversion was indeed complete and we use therefore only the EOS of quark matter for solving the diffusion equation. While introducing a layer of unburned nucleonic matter would not alter substantially the initial luminosity of the neutrino signal (at low densities the mean free paths of quark matter and nucleonic matter are both large and neutrinos are almost untrapped), the further conversion of the layer of the unburned material represents a source of energy which will necessarily prolong the neutrino signal. A model for the burning of the nucleonic material left after the hydrodynamical combustion would be needed.

Finally, we must specify the neutrino mean free paths. As explained before, for the system we are investigating here, cooling is likely to be the most important process, while the deleptonization (or the releptonization) should be subdominant. Within this assumption the most important reaction between neutrinos and quark matter is the scattering of nondegenerate neutrinos whose inverse mean free path reads [52]

$$
\frac{\sigma_{S}}{V}=\frac{G_{F}^{2} E_{\nu}^{3} \mu_{i}^{2}}{5 \pi^{3}}
$$

where $G_{F}=1.17 \mathrm{GeV}^{-2}$ is the Fermi constant, $E_{\nu}$ is the energy of the neutrino or antineutrino and $\mu_{i}$ is the chemical potential of the particle involved in the scattering (up, down, or strange quarks). Here, instead of calculating the spectral averages of the mean free paths, we evaluate the mean free path at $E_{\nu}=\pi T$, i.e., at the mean energy of neutrinos in thermal equilibrium.

With this setup we can now numerically solve Eqs. (1)-(5) (see [32] for the discussion of the algorithm used). Results for the temperature profiles as functions of the fraction of enclosed baryonic mass (the enclosed baryonic mass $a$ over the total baryonic mass $M_{B}$ ) are shown in Figs. 3 and 4 for the 1.4 and $1.8 M_{\odot}$ cases, respectively. The initial temperature profile presents a steep gradient as resulting from the combustion simulations. This steep gradient leads, within the diffusion approximation we are adopting here, to a strong flux of heat which rapidly smoothes the temperature profile and after a few seconds the central temperature drops to half of its initial value. After $\sim 10$ s and $\sim 15 \mathrm{~s}$ in the two cases, respectively, the star has reached a uniform temperature of about $1 \mathrm{MeV}$ and neutrinos can freely stream within the star, the standard long term cooling sets in at this point. Notice that the higher the mass of the star, the longer the time needed to cool the star. The most 


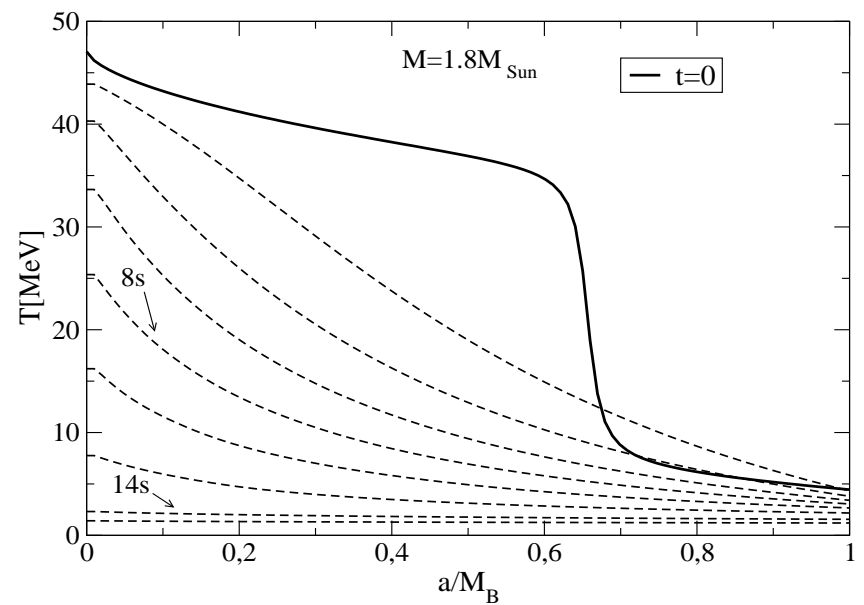

FIG. 4: Temperature evolution as a function of enclosed baryonic mass for a $1.8 M_{\odot}$ star.

important result of this paper is shown in Fig. 5, where we display the total neutrino and antineutrino (summed over the flavors) luminosity as a function of time. The luminosity is calculated as [48]

$$
L=-\left.e^{2 \phi} 4 \pi r^{2} \frac{\lambda_{\epsilon_{\nu}}}{3} \frac{\partial \epsilon_{\nu}}{\partial r}\right|_{r=R}
$$

Starting with an initial value of $\sim 3 \times 10^{52} \mathrm{erg} / \mathrm{s}$, it drops after $10-15 \mathrm{~s}$ to $10^{50} \mathrm{erg}$. The initial value of the luminosity is very similar to the typical values of protoneutron stars thus making the process studied here as interesting as protoneutron stars from the phenomenological point of view. The obtained signal lasts, however, shorter by a factor $3-4$ than the protoneutron star signal (for which the luminosity of $10^{50} \mathrm{erg}$ is reached after about $60 \mathrm{~s})$. The shorter evolution is attributed to different aspects: i) The neutrino mean free paths in quark matter are larger than in nucleonic matter [52], and ii) in protoneutron stars the deleptonization process mentioned before significantly contributes to the emission. This study represents a first estimate of the neutrino signal emitted after the conversion of a neutron star into a strange star. Clearly, more detailed studies with a better treatment of the neutrino transport, an EOS extended to nonbeta stable matter to include the lepton number conservation during the burning and its transport within the star after the formation of quark matter will improve the estimates. As explained before, the burning of the material left after the hydrodynamical combustion could represent an important source of energy which would then prolong the neutrino emission. In [53] one-dimensional

hydrodynamic simulations of the combustion flame, including neutrino emission and strange quark diffusion have been conducted: It turns out that neutrino cooling can halt the burning interface. It would be interesting to extend these calculations by use of a three-dimensional

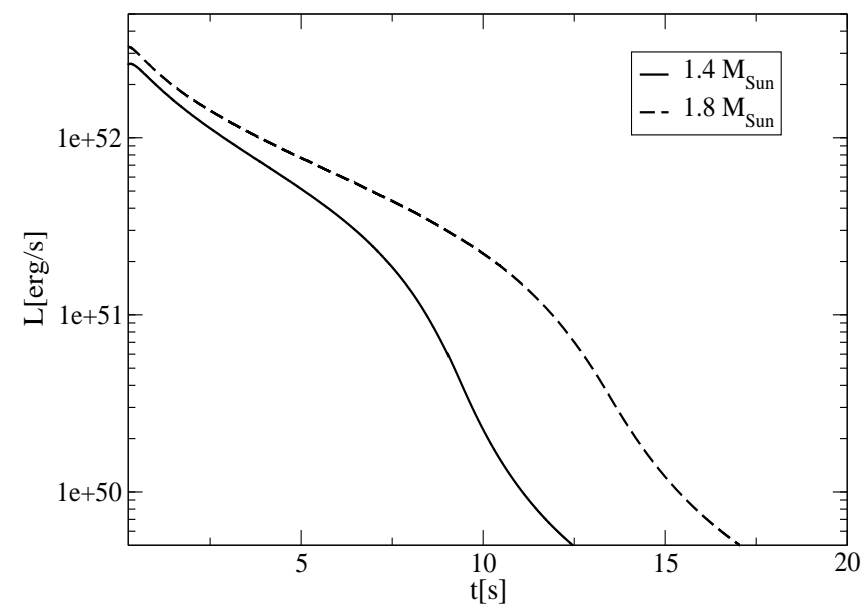

FIG. 5: Total neutrino and antineutrino luminosity as a function of time.

hydrodynamic code. Finally, we note that there are substantial uncertainties in the models usually adopted for describing the high density nuclear matter equation of state. These affect the results displayed in Fig. 5, which are to be taken as an order of magnitude calculation. It is, however, interesting to note that the estimate of the total energy released in the conversion, $\sim 10^{53} \mathrm{erg}$, is consistent with other estimates obtained by using equations of state for nuclear matter including hyperons; see, for instance, [12].

\section{DISCUSSION AND CONCLUSIONS}

From the previous results on the neutrino luminosities one can easily estimate the total energy released by the conversion process to be of the order of $10^{53} \mathrm{erg}$. It is thus powerful enough to be compared with the energy released within the most violent and, to some extent, still mysterious explosions of the Universe, i.e., SNe and long GRBs. It is then clearly tempting to associate at least some of these explosions to the formation of a strange star (the first proposals about this connection were presented many years ago [54, 55], see also the more recent Refs. 10, 56, 57] ). In particular, the formation of strange quark matter (regardless of its absolute stability) could provide an additional energy injection which triggers the explosion of core collapse supernovae [58 63]. None of the presently available simulations could produce explosions for high mass progenitors (with masses larger than $\sim 20 M_{\odot}$ ), and the possible appearance of quark matter could help in solving this problem.

Apart from the energy released in the birth of a strange star, also the temporal delay with respect to the collapse of the progenitor star and the birth of the neutron star could possibly explain some of the puzzling observations connected to SNe and GRBs. In Ref. [54] a two- 
neutrino-burst scenario is proposed for the neutrino signal of SN1987A: the data of the LSD detector would indicate a burst of neutrinos that occurred $\sim 5 \mathrm{~h}$ before the well-known K2, IMB and Baksan neutrino events. The second burst, suggests the author, could be, for instance, associated with the birth of a strange star.

It is widely accepted that long GRBs are phenomena connected with the collapse of massive stars and that they are intimately connected to SNe. In some cases, a sizable temporal delay (ranging from hours to years) between a SN and the subsequent GRB was inferred from the data (see [10]). In those cases the second explosive event, i.e. the GRB, could be associated with the conversion of a neutron star into a star containing quark matter as proposed in [10].

The puzzling observations mentioned before (the LSD neutrino signal and the long time delay between SN and GRB) have been, however, under debate for many years and none of them is considered to be statistically robust. Therefore they do not provide a clear proof of the existence of quark matter in astrophysical systems. A more direct and clean analysis can instead be performed just by considering the light curves of the prompt emissions of GRBs: It seems again that, at least in some cases, after a first burst a second burst occurs which is delayed by up to hundreds of seconds with respect to the first 64]. Between the two bursts a quiescent time is present during which it is likely that the inner engine is not active. In [64], by performing a statistical analysis using the sample of GRB light curves of the BATSE satellite, hints are presented in favor of the interpretation of long quiescent times as periods during which the inner engine is indeed dormant. A spectacular event of this type was recently detected by the Swift satellite 65]: The second burst is 11 min delayed with respect to the first one. Such a long quiescent time challenges popular models for the GRB inner engine, i.e., the collapsar model [66] and the protomagnetar model [24]. In [65], the following scenario is proposed: The first burst is generated by a rapidly rotating magnetar, and the second burst is due to a delayed collapse of the star into a black hole (if enough mass accretes onto the magnetar, about $\left.1 M_{\odot}\right)$. Recent numerical simulations of the accretion induced collapse of a neutron star indicate, however, that these events could be sources of short GRBs instead of the long ones 67]. Here we speculate that those double bursts could be instead related to the conversion of a neutron star to a strange star. Within the protomagnetar model of long GRBs, the source of energy is provided by the ro- tational energy of the star and for the prompt emission, in addition to the spin down rate, also the neutrino wind released by the hot surface of the star is crucial: A high neutrino luminosity implies a large value for the mass loss rate which inhibits the mechanism at the origin of the gamma radiation. Only when the neutrino luminosity drops to a critical value, in the untrapping regime, is the prompt emission of the GRB realized. The strange star at birth could then, in principle, generate a new burst. The quiescent time would correspond, in this scenario, to the time needed to trigger the conversion process (for instance, because of the spinning down, the central density increases and at some point nucleation can start as computed in [68]). A detailed numerical study of this possibility is an important outlook of this work.

Clearly, the main motivation of this paper is to show that the conversion process of a neutron star into a strange star generates a strong neutrino signal which is relevant from the phenomenological point of view. Being the first quantitative study in this problem, several assumptions had to be adopted. For studying quantitatively the phenomenological consequences of this process it is important to improve our calculation especially by introducing the chemical potential of electron neutrinos and the lepton number diffusion equation. Modeling the burning of the material left after the combustion would also be important for obtaining a better estimate of the duration of the neutrino signal.

Finally, testing our theoretical results by means of a direct neutrino detection is clearly very difficult: If such processes really occur in the Universe, their rate is probably significantly lower than that of core collapse SN events, therefore making a detection highly improbable. On the other hand, we have plenty of data on long GRBs: We have suggested that these data already contain some interesting information which would possibly indicate that strange quark matter is really formed in compact stellar objects.

We thank A. Drago for valuable discussions. G.P. acknowledges financial support from the Italian Ministry of Research through the program "Rita Levi Montalcini". The work of F.K.R. is supported by the Deutsche Forschungsgemeinschaft (DFG) via the Emmy Noether Program (RO 3676/1-1), and by the ARCHES prize of the German Ministry of Education and Research (BMBF).
[1] N. Itoh, Prog.Theor.Phys. 44, 291 (1970).

[2] A. Bodmer, Phys.Rev. D4, 1601 (1971).

[3] E. Witten, Phys. Rev. D 30, 272 (1984).

[4] P. Haensel, J. Zdunik, and R. Schaeffer, Astron.Astrophys. 160, 121 (1986).

[5] C. Alcock, E. Farhi, and A. Olinto, Astrophys.J. 310,
261 (1986).

[6] A. Bauswein, H.-T. Janka, R. Oechslin, G. Pagliara, I. Sagert, et al., Phys.Rev.Lett. 103, 011101 (2009), 0812.4248 .

[7] A. Bauswein, R. Oechslin, and H.-T. Janka, Phys.Rev. D81, 024012 (2010), 0910.5169. 
[8] M. L. Olesen and J. Madsen, Phys.Rev. D49, 2698 (1994), astro-ph/9401002.

[9] K. Iida and K. Sato, Phys.Rev. C58, 2538 (1998), nuclth/9808056.

[10] Z. Berezhiani, I. Bombaci, A. Drago, F. Frontera, and A. Lavagno, Astrophys.J. 586, 1250 (2003), astro$\mathrm{ph} / 0209257$.

[11] A. Drago, A. Lavagno, and G. Pagliara, Phys.Rev. D69, 057505 (2004), nucl-th/0401052.

[12] I. Bombaci, I. Parenti, and I. Vidana, Astrophys.J. 614, 314 (2004), astro-ph/0402404.

[13] I. Bombaci, G. Lugones, and I. Vidana, Astron.Astrophys. 462, 1017 (2007), astro-ph/0603644.

[14] B. Mintz, E. Fraga, G. Pagliara, and J. Schaffner-Bielich, Phys.Rev. D81, 123012 (2010), 0910.3927.

[15] I. Bombaci, D. Logoteta, P. Panda, C. Providencia, and I. Vidana, Phys.Lett. B680, 448 (2009), 0910.4109.

[16] D. Logoteta, C. Providencia, I. Vidana, and I. Bombaci, Phys.Rev. C85, 055807 (2012), 1204.5909.

[17] A. V. Olinto, Physics Letters B 192, 71 (1987).

[18] J. Horvath and O. Benvenuto, Phys.Lett. B213, 516 (1988).

[19] G. Lugones, C. Ghezzi, E. de Gouveia Dal Pino, and J. Horvath, Astrophys.J. 581, L101 (2002), astro$\mathrm{ph} / 0207262$.

[20] H. Cho, K. Ng, and A. Speliotopoulos, Phys.Lett. B326, 111 (1994), astro-ph/9305006.

[21] A. Drago, A. Lavagno, and I. Parenti, Astrophys.J. 659, 1519 (2007), astro-ph/0512652.

[22] M. Herzog and F. K. Röpke, Phys.Rev. D84, 083002 (2011), 1109.0539.

[23] P. Keranen, R. Ouyed, and P. Jaikumar, Astrophys.J. 618, 485 (2004), astro-ph/0406448.

[24] B. Metzger, D. Giannios, T. Thompson, N. Bucciantini, and E. Quataert (2010), 1012.0001.

[25] P. Demorest, T. Pennucci, S. Ransom, M. Roberts, and J. Hessels, Nature 467, 1081 (2010), 1010.5788.

[26] J. M. Lattimer and F. D. Swesty, Nuclear Physics A 535, 331 (1991).

[27] H. Shen, H. Toki, K. Oyamatsu, and K. Sumiyoshi, Progress of Theoretical Physics 100, 1013 (1998), arXiv:nucl-th/9806095.

[28] E. Farhi and R. Jaffe, Phys.Rev. D30, 2379 (1984).

[29] E. S. Fraga, R. D. Pisarski, and J. Schaffner-Bielich, Phys.Rev. D63, 121702 (2001), hep-ph/0101143.

[30] M. G. Alford, A. Schmitt, K. Rajagopal, and T. Schafer, Rev.Mod.Phys. 80, 1455 (2008), 0709.4635.

[31] G. W. Carter and S. Reddy, Phys.Rev. D62, 103002 (2000), hep-ph/0005228.

[32] G. Pagliara, Phys.Rev. D83, 125013 (2011), 1012.3046.

[33] S. Weissenborn, I. Sagert, G. Pagliara, M. Hempel, and J. Schaffner-Bielich, Astrophys.J. 740, L14 (2011), 1102.2869 .

[34] M. Alford, M. Braby, M. Paris, and S. Reddy, Astrophys.J. 629, 969 (2005), nucl-th/0411016.

[35] P. Colella and P. R. Woodward, J.Comput.Phys. 54, 174 (1984).

[36] M. Reinecke, W. Hillebrandt, J. C. Niemeyer, R. Klein, and A. Gröbl, Astron.Astrophys. 347, 724 (1999), arXiv:astro-ph/9812119.

[37] M. Reinecke, W. Hillebrandt, and J. C. Niemeyer, Astron.Astrophys. 391, 1167 (2002), arXiv:astro$\mathrm{ph} / 0206459$.

[38] F. K. Röpke and W. Hillebrandt, Astron.Astrophys. 431,
635 (2005), arXiv:astro-ph/0409286.

[39] A. Bhattacharyya, S. K. Ghosh, R. Mallick, and S. Raha, Phys.Rev. C76, 052801 (2007), 0707.3677.

[40] B. Niebergal, R. Ouyed, and P. Jaikumar, Phys. Rev. C 82, 062801 (2010), 1008.4806.

[41] W. Schmidt, J. C. Niemeyer, and W. Hillebrandt, Astron.Astrophys. 450, 265 (2006), arXiv:astro$\mathrm{ph} / 0601499$.

[42] W. Schmidt, J. C. Niemeyer, W. Hillebrandt, and F. K. Röpke, Astron.Astrophys. 450, 283 (2006), arXiv:astro$\mathrm{ph} / 0601500$.

[43] L. Hudepohl, B. Muller, H.-T. Janka, A. Marek, and G. Raffelt, Phys.Rev.Lett. 104, 251101 (2010), 0912.0260

[44] T. Fischer, S. Whitehouse, A. Mezzacappa, F.-K. Thielemann, and M. Liebendorfer, Astron.Astrophys. 517, A80 (2010), 0908.1871.

[45] A. Burrows and J. M. Lattimer, Astrophys. J. 307, 178 (1986).

[46] W. Keil and H.-T. Janka, Astron. Astrophys. 296, 145 (1995).

[47] M. Prakash, I. Bombaci, M. Prakash, P. J. Ellis, J. M. Lattimer, et al., Phys.Rept. 280, 1 (1997), nuclth/9603042.

[48] J. Pons, S. Reddy, M. Prakash, J. Lattimer, and J. Miralles, Astrophys.J. 513, 780 (1999), astro-ph/9807040.

[49] J. A. Pons, J. A. Miralles, M. Prakash, and J. M. Lattimer, Astrophys.J. 553, 382 (2001), astro-ph/0008389.

[50] J. A. Pons, A. W. Steiner, M. Prakash, and J. M. Lattimer, Phys.Rev.Lett. 86, 5223 (2001), astro$\mathrm{ph} / 0102015$.

[51] G. Lugones, O. Benvenuto, and H. Vucetich, Phys.Rev. D50, 6100 (1994).

[52] A. W. Steiner, M. Prakash, and J. M. Lattimer, Phys.Lett. B509, 10 (2001), astro-ph/0101566.

[53] B. Niebergal, R. Ouyed, and P. Jaikumar, Phys.Rev. C82, 062801 (2010), 1008.4806.

[54] A. De Rujula, Phys.Lett. B193, 514 (1987).

[55] K. Cheng and Z. Dai, Phys.Rev.Lett. 77, 1210 (1996), astro-ph/9510073.

[56] I. Bombaci and B. Datta, Astrophys.J. 530, L69 (2000), astro-ph/0001478.

[57] R. Ouyed and F. Sannino, Astron.Astrophys. 387, 725 (2002), astro-ph/0103022.

[58] N. Gentile, M. Aufderheide, G. Mathews, F. Swesty, and G. Fuller, Astrophys.J. 414, 701 (1993).

[59] A. Drago and U. Tambini, J.Phys. G25, 971 (1999), astro-ph/9703138.

[60] I. Sagert, T. Fischer, M. Hempel, G. Pagliara, J. Schaffner-Bielich, et al., Phys.Rev.Lett. 102, 081101 (2009), 0809.4225.

[61] T. Fischer, I. Sagert, G. Pagliara, M. Hempel, J. Schaffner-Bielich, et al., Astrophys.J.Suppl. 194, 39 (2011), 1011.3409.

[62] A. Drago, G. Pagliara, G. Pagliaroli, F. L. Villante, and F. Vissani, AIP Conf.Proc. 1056, 256 (2008), 0809.0518.

[63] G. Pagliara, M. Hempel, and J. Schaffner-Bielich, Phys.Rev.Lett. 103, 171102 (2009), 0907.3075.

[64] A. Drago and G. Pagliara, Astrophys.J. 665, 1227 (2007), astro-ph/0512602.

[65] B.-B. Zhang, D. N. Burrows, B. Zhang, P. Meszaros, G. Stratta, et al., Astrophys.J. 748, 132 (2012), 1111.2922 .

[66] S. E. Woosley, Astrophys.J. 405, 273 (1993). 
[67] B. Giacomazzo and R. Perna, Astrophys.J. 758, L8 (2012), 1209.0783.

[68] N. Yasutake, M.-a. Hashimoto, and Y. Eriguchi, Prog.Theor.Phys. 113, 953 (2005), astro-ph/0411434.

[69] In principle, absorption processes of neutrinos by down quarks could lead to a "re-leptonization" of the star, i.e. a temporal window in which, probably within the outer layers of the star, matter changes its chemical composition. We retain this possibility as a future development of this work. 